\title{
Lymphoid tissue-resident Alcaligenes LPS induces IgA production without excessive inflammatory responses via weak TLR4 agonist activity
}

\author{
Naoko Shibata ${ }^{1,2,3}$, Jun Kunisawa ${ }^{1,3,4,5,6}$, Koji Hosomi ${ }^{3}$, Yukari Fujimoto ${ }^{7,8}$, Keisuke Mizote ${ }^{8}$, \\ Naohiro Kitayama $^{8}$, Atsushi Shimoyama ${ }^{8}$, Hitomi Mimuro, ${ }^{9,10}$, Shintaro Sato ${ }^{1,4,11}$, Natsuko Kishishita ${ }^{12}$, \\ Ken J Ishii ${ }^{12,13}$, Koichi Fukase ${ }^{8}$ and Hiroshi Kiyono ${ }^{1,2,4}$
}

\begin{abstract}
Alcaligenes are opportunistic commensal bacteria that reside in gut-associated lymphoid tissues such as Peyer's patches (PPs); however, how they create and maintain their homeostatic environment, without inducing an excessive inflammatory response remained unclear. We show here that Alcaligenes-derived lipopolysaccharide (Alcaligenes LPS) acts as a weak agonist of toll-like receptor 4 and promotes IL-6 production from dendritic cells, which consequently enhances IgA production. The inflammatory activity of Alcaligenes LPS was weaker than that of Escherichia coli-derived LPS and therefore no excessive inflammation was induced by Alcaligenes LPS in vitro or in vivo. Alcaligenes LPS also showed adjuvanticity, inducing antigen-specific immune responses without excessive inflammation. These findings reveal the presence of commensal bacteria-mediated homeostatic inflammatory conditions within PPs that produce optimal IgA induction without causing pathogenic inflammation and suggest that Alcaligenes LPS could be a safe and potent adjuvant.
\end{abstract}

\section{INTRODUCTION}

Intestinal commensal bacteria affects the development and function of the host immune system, including the production of secretory $\operatorname{IgA}(\operatorname{SIgA})^{1}$ and the development of intraepithelial $\mathrm{T}$ lymphocytes. ${ }^{2}$ Accumulating evidence has revealed that particular kinds of commensal bacteria control the differentiation of specific T-cell populations. For example, segmented filamentous bacteria induce the differentiation of Th17 cells ${ }^{3}$ and clostridial strains can induce regulatory $\mathrm{T}$ cells. ${ }^{4}$

Although these studies mainly focused on the commensal bacteria in the intestinal lumen or mucus layers, genome-based bacterial analysis using intestinal tissue allowed us to identify Alcaligenes as symbiotic resident bacteria of Peyer's patches (PPs), a major gut-associated lymphoid tissue in the small intestine. ${ }^{5}$ Oral inoculation of Alcaligenes into germ-free mice resulted in the sustained growth of Alcaligenes in the PPs, suggesting that at least some portion of the inoculum survived in the PPs. ${ }^{5}$ Our subsequent study showed that lymphoid tissue-resident commensal (LRC) bacteria including Alcaligenes have a greater ability to survive in dendritic cells (DCs) than do luminal-resident bacteria (e.g., Escherichia coli [E. coli]) and modulate the production of cytokines such as IL-1 $\beta$,

${ }^{1}$ Division of Mucosal Immunology, Department of Microbiology and Immunology, The Institute of Medical Science, The University of Tokyo, Tokyo, Japan. ${ }^{2}$ Department of Immunology, Graduate School of Medicine, Chiba University, Chiba, Japan. ${ }^{3}$ Laboratory of Vaccine Materials and Laboratory of Gut Environmental System, National Institutes of Biomedical Innovation, Health and Nutrition (NIBIOHN), Osaka, Japan. ${ }^{4}$ International Research and Development Center for Mucosal Vaccines, The Institute of Medical Science, The University of Tokyo, Tokyo, Japan. ${ }^{5}$ Graduate School of Medicine, Graduate School of Pharmaceutical Sciences, Graduate School of Dentistry, Osaka University, 1-1 Yamadaoka, Suita-city, Osaka, Japan. ${ }^{6}$ Department of Microbiology and Immunology, Kobe University Graduate School of Medicine, Kobe-city, Hyogo, Japan. ${ }^{7}$ Department of Chemistry, Faculty of Science and Technology, Keio University, Kanagawa, Japan. ${ }^{8}$ Department of Chemistry, Graduate School of Science, Osaka University, Osaka, Japan. ${ }^{9}$ Division of Bacteriology, Department of Infectious Diseases Control, International Research Center for Infectious Diseases, Institute of Medical Science, The University of Tokyo, Tokyo, Japan. ${ }^{10}$ Department of Infection Microbiology, Research Institute for Microbial Diseases, Osaka University 3-1, Yamadaoka, Osaka, Japan. ${ }^{11}$ Mucosal Vaccine Project, BIKEN Innovative Vaccine Research Alliance Laboratories, Research Institute for Microbial Diseases, Osaka University, Osaka, Japan. ${ }^{12}$ Laboratory of Adjuvant Innovation, National Institutes of Biomedical Innovation, Health and Nutrition (NIBIOHN), Osaka, Japan and ${ }^{13}$ Laboratory of Vaccine Science, WPI Immunology Frontier Research Center (IFReC), Osaka University, Osaka, Japan. Correspondence: J Kunisawa (kunisawa@nibiohn.go.jp); H Kiyono (kiyono@ims.u-tokyo.ac.jp) 
IL-6, IL-10, IL-12p40, and IL-23 from DCs. ${ }^{6} \mathrm{We}$ also demonstrated that not only live bacteria, but also heat-killed Alcaligenes induces the production of cytokines such as IL-6, BAFF, and TGF- $\beta$ from DCs, key cytokines in the enhancement of IgA production. ${ }^{5}$ Collectively, germ-free mice monoassociated with LRC bacteria (e.g., Alcaligenes and Bordetella) induced IL-22-producing type 3 innate lymphoid cells in the PPs and an increase in SIgA production in the feces. ${ }^{5,6}$ Both type 3 innate lymphoid cells and IgA antibodies are thought to have an important role in maintaining the restricted distribution of Alcaligenes inside PPs. ${ }^{5,7}$

Although these findings revealed the involvement of lymphoid tissue-resident Alcaligenes in the development and regulation of the host immune system, how Alcaligenes establishes symbiosis without inducing excessive inflammatory responses in the PPs and maintains this homeostatic immunological condition remains unknown. In this study, we focused on LPS, a major component of the outer membrane of gramnegative bacteria like Alcaligenes. It is well known that the structure of LPS differs among the gram-negative bacteria, and this difference determines the degree of toll-like receptor 4 (TLR4)-mediated inflammatory activity. ${ }^{8,9}$ In this study, we found that Alcaligenes-derived lipopolysaccharide (Alcaligenes LPS) acts as a weak TLR4 agonist and thus creates a homeostatic inflammatory condition that includes IgA responses in PPs without the excessive pathological inflammation that is generally caused by classical LPS. These findings suggest Alcaligenes LPS could be used as a safe and effective vaccine adjuvant.

\section{RESULTS}

\section{Detection of Alcaligenes in the DCs of the subepithelial dome region of PPs}

Our previous study demonstrated that Alcaligenes are initially taken up by $\mathrm{M}$ cells located in the follicle-associated epithelium of PPs. ${ }^{10}$ Building on these results, we sought to elucidate the distribution of the commensal bacteria inside PPs, including examining the possibility of intracellular habitation since the region underneath the follicle-associated epithelium, known as the subepithelial dome region, is enriched with antigenpresenting cells. ${ }^{11}$ To examine the microlocalization of Alcaligenes in the PPs, we initially performed an intestinal loop assay with GFP-expressing Alcaligenes (GFP-Alcaligenes). Histological analysis revealed that Alcaligenes were predominantly located in $\mathrm{CD}_{11 \mathrm{c}^{+}} \mathrm{DCs}$ in the subepithelial dome region of the PPs (Figure 1a). To confirm this finding, we performed fluorescent in situ hybridization analysis using CD11c diphtheria toxin (DT) receptor transgenic (CD11cDTR tg) mice. Alcaligenes were detected in the PPs of mocktreated CD11c-DTR tg mice, whereas limited signals derived from Alcaligenes were detected in the absence of DCs in the DTtreated CD11c-DTR tg mice (Figure $\mathbf{1 b}$ and Supplementary Figure S1 online). We further showed that the uptake efficiency of Alcaligenes was higher than that of E. coli (Figure 1c). These data indicate that DCs are the primary residential site for the preferential and efficient retention of Alcaligenes in the subepithelial dome region of PPs.

\section{Heat-killed Alcaligenes have low inflammatory activity}

We next examined the inflammatory properties of Alcaligenes against DCs. DCs were cultured with heat-killed Alcaligenes or E. coli as a control, and IL-6 production in the culture supernatant measured. Heat-killed Alcaligenes promoted IL-6 production from bone marrow-derived DCs in a dosedependent manner, but its ability was weaker than that of heat-killed E. coli (Figure 2a). The IL-6 50\% effective dose $\left(\mathrm{ED}_{50}\right.$ value $)$ of heat-killed Alcaligenes was 30 times that of heatkilled E. coli (Figure 2b). Similar results were obtained with DCs isolated from PPs (Figure 2c). To exclude the possibility that the reduced amounts of IL- 6 were owing to Alcaligenesmediated cell death, we confirmed that the viability of the DCs treated with heat-killed Alcaligenes was comparable to that of the DCs treated with E. coli (Figure 2d). These results suggest that Alcaligenes are less effective than E. coli at inducing inflammatory cytokines such as IL-6.

In addition to inflammatory cytokines (e.g., IL-6), bacteria also induce the production of the inhibitory cytokine IL-10, a

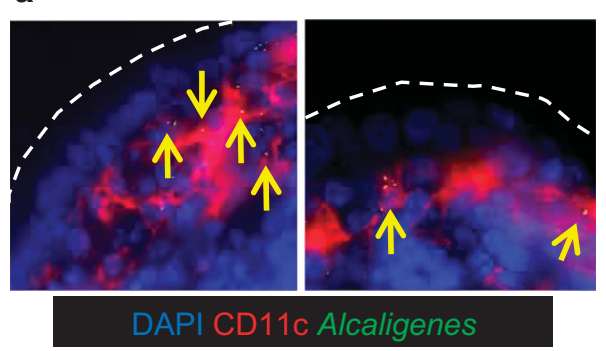

b

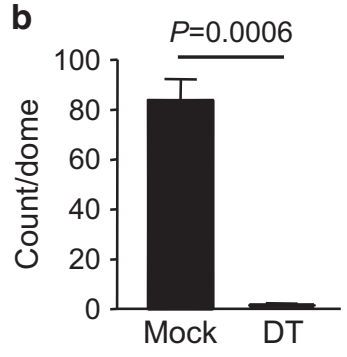

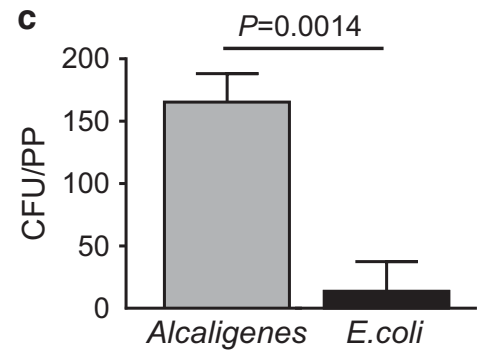

Figure 1 Detection of Alcaligenes in the DCs of the subepithelial dome region of PPs. (a) GFP-expressing Alcaligenes was injected into an intestinal loop containing a single PP. After a 2-h incubation, the PP was isolated for immunohistological analysis with CD11c-specific Ab (red) and DAPI (blue). Arrows indicate Alcaligenes in the DCs. Two representative pictures are shown; similar results were obtained from three independent experiments. (b) CD11c-DTR tg mice were intraperitoneally injected with DT or PBS (mock). After $24 \mathrm{~h}$, PPs were isolated for whole-mount fluorescence in situ hybridization analysis. The presence of Alcaligenes was visually identified with an Alcaligenes-specific probe (ALBO). The number of fluorescent dots per dome of PP was calculated. Data are representative of three independent experiments (three mice per group for each experiment). DT, diphtheria toxin. (c) GFP-expressing Alcaligenes or E.coli was injected into an intestinal loop containing a single PP. After a 2-h incubation, the PP was isolated to measure the bacterial uptake efficiency. Data are representative of two independent experiments (three mice per group for each experiment). 


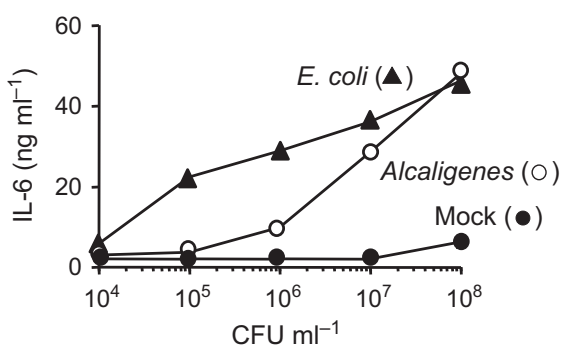

b

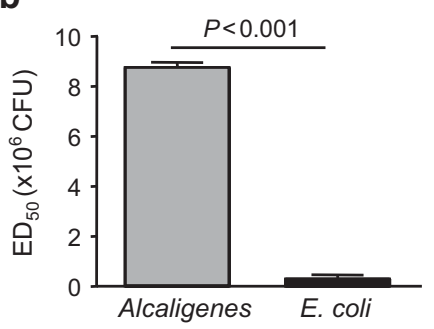

C

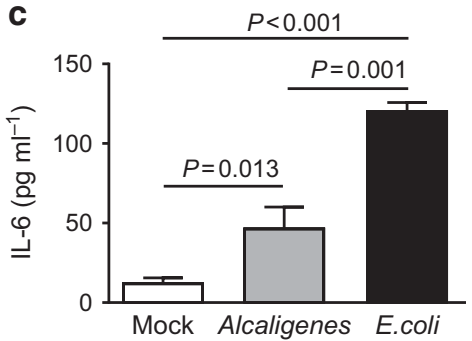

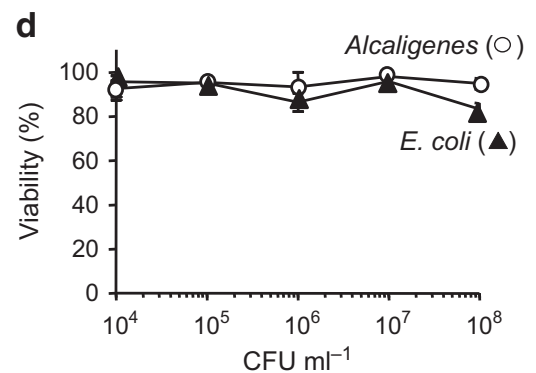

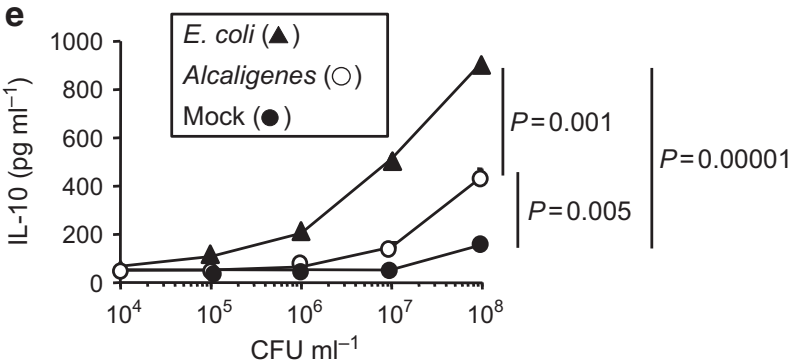

Figure 2 Heat-killed Alcaligenes induce low levels of cytokine production from DCs. BMDCs $(\mathbf{a}, \mathbf{b}, \mathbf{d}, \mathbf{e})$ or DCs purified from the PPs $(\mathbf{c})\left(1 \times 10^{5}\right.$ cells per well) were cultured with various concentrations of heat-killed $E$. coli, heat-killed Alcaligenes, or PBS (mock) for $48 \mathrm{~h}$ ( $n=3$ per group). (a, c, d) Culture

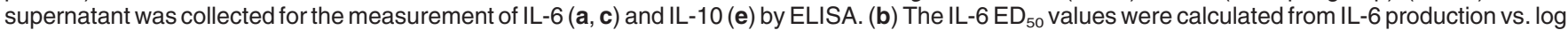
concentration curves. (d) Cell viability was determined by using the trypan blue exclusion assay. Data are representative of three independent experiments. $\mathrm{ED}_{50}$, effective dose 50 .

which prevents the production of inflammatory cytokines by inhibiting the activation of nuclear factor $\kappa \mathrm{B}$, a transcription factor involved in inflammatory cytokine gene expression. ${ }^{12}$ We therefore measured the production of IL-10 from DCs cultured with heat-killed Alcaligenes or E. coli. IL-10 production induced by heat-killed Alcaligenes was weaker than that induced by heat-killed E. coli (Figure 2e). These findings suggest that the decreased production of IL- 6 from DCs cultured with heat-killed Alcaligenes was not owing to IL-10mediated inhibition. Together, these results demonstrate that Alcaligenes are less effective than E. coli at inducing both inflammatory and inhibitory cytokines, at least in the heatkilled form.

\section{Alcaligenes LPS acts as a weak TLR4 agonist}

LPS is a major biologically active component of the outer membrane of gram-negative bacteria including Alcaligenes and E. coli. ${ }^{8,9}$ Several lines of evidence have demonstrated that the inflammatory activity of LPS differs among bacteria. ${ }^{8}$ We found that depletion of LPS from Alcaligenes by using alkaline treatment decreased its ability to enhance IL- 6 production from DCs (Figure 3a). These findings led us to examine the inflammatory activity of Alcaligenes LPS compared with that of E. coli. We measured the amount of LPS isolated from the same number of Alcaligenes and E. coli and found that more LPS was isolated from Alcaligenes than from E. coli (Supplementary Figure S2a). In contrast, the viability of DCs treated with Alcaligenes LPS was identical to that of DCs treated with E. coli LPS (Supplementary Figure S2b). Together with the heat-killed Alcaligenes data (Figure 2), these results indicate that the inflammatory activity of heat-killed Alcaligenes is weak even though the amount of LPS per cell is greater than that of E. coli.

Using LPS isolated from Alcaligenes and E. coli, we next compared the production of IL-6 from DCs cultured with the LPS from these two microorganisms. In agreement with our findings regarding heat-killed Alcaligenes (Figure 2a), Alcaligenes LPS-treated DCs induced a lower level of IL-6 production than did DCs treated with E. coli LPS (Figure 3b). The IL-6 $\mathrm{ED}_{50}$ value of Alcaligenes LPS was 30 times that of $E$. coli LPS (Figure 3c). Similar results were obtained with DCs isolated from PPs (Figure 3d). Similar to pathogenic E. coli (O111), LPS derived from commensal E. coli (S17) was better able to induce IL- 6 production than was Alcaligenes LPS (Supplementary Figure S3). Furthermore, Alcaligenes LPStreated DCs induced a lower level of TNF- $\alpha$ than did DCs treated with E. coli LPS (Supplementary Figure S4). There was also no difference in IL-10 production from DCs with Alcaligenes LPS compared with those treated with E. coli LPS (Figure 3e). These data are in agreement with the results from heat-killed bacteria (Figure 2) and indicate that Alcaligenes LPS has a weak biological activity and thus induce lower levels of IL- 6 and TNF- $\alpha$ from DCs relative to those induced by E. coli LPS.

LPS derived from certain gram-negative bacteria, such as Rhodobacter sphaeroides and Porphyromonas gingivalis, can act as an antagonist or a competitive inhibitor of other types of LPS. ${ }^{13,14}$ To test the possibility whether Alcaligenes LPS acts as an antagonist and thus competitively inhibits the activity of E. coli LPS, DCs were cultured with E. coli LPS in the presence of different amounts of Alcaligenes LPS. When DCs were cocultured with a small amount of E. coli LPS and excess 
a

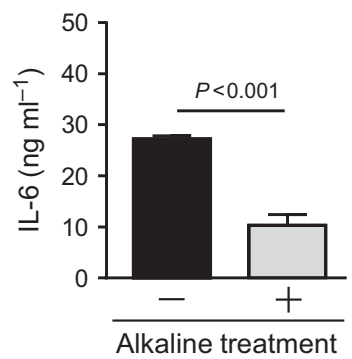

d

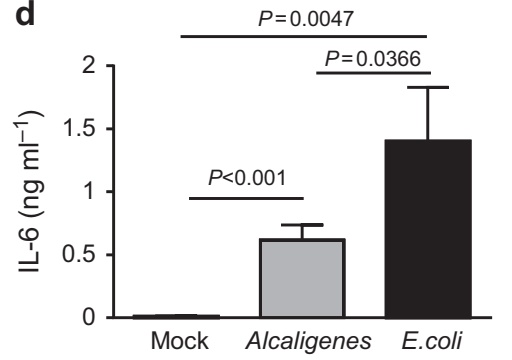

b

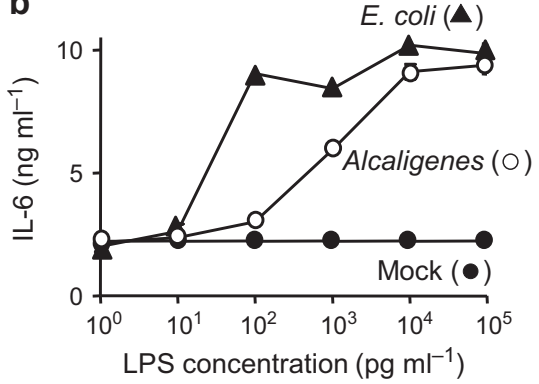

e

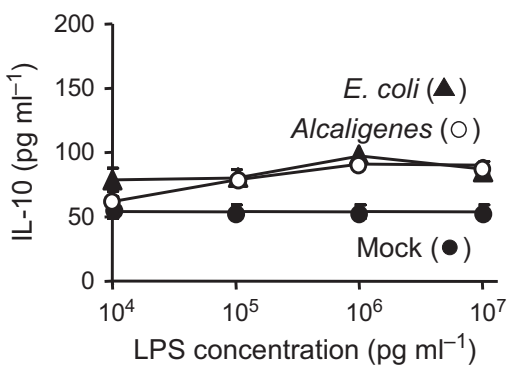

c

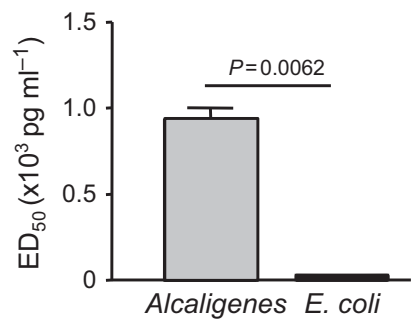

f

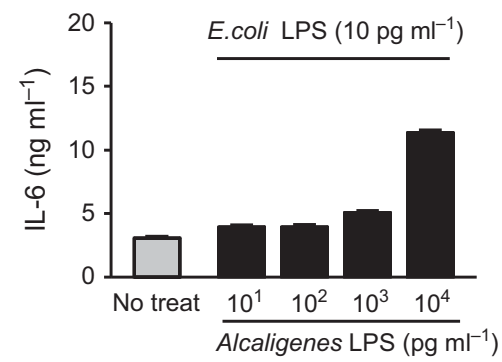

Figure 3 Alcaligenes LPS induces low levels of IL-6 production from DCs. (a) BMDCs $\left(1 \times 10^{5}\right.$ cells per well) were cultured with alkaline-treated, or mock-treated, heat-killed Alcaligenes ( $10^{6} \mathrm{CFU}$ per well) for $48 \mathrm{~h}$ ( $n=3$ per group). Culture supernatant was collected to measure IL-6 by means of an ELISA. Data are representative of two independent experiments. BMDCs $(\mathbf{b}, \mathbf{e}, \mathbf{f})$ or DCs purified from the PPs (d) $\left(1 \times 10^{5}\right.$ cells per well) were cultured with various concentrations (b,e, f) or $10^{4} \mathrm{pg}$ (d) of LPS per well from E. colior Alcaligenes, or were mock-treated (PBS) for $48 \mathrm{~h}$ ( $n=3$ per group). (b, $\left.\mathbf{d}, \mathbf{e}\right)$

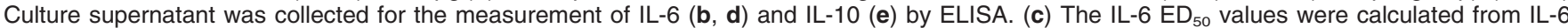
production vs. log concentration curves. (f) BMDCs were cultured with $10 \mathrm{pg} \mathrm{ml}^{-1}$ E. coliLPS in the presence of various concentrations of Alcaligenes LPS for $48 \mathrm{~h}$. Culture supernatant was collected for the measurement of IL-6 by ELISA ( $n=3$ per group). Data are representative of two independent experiments.
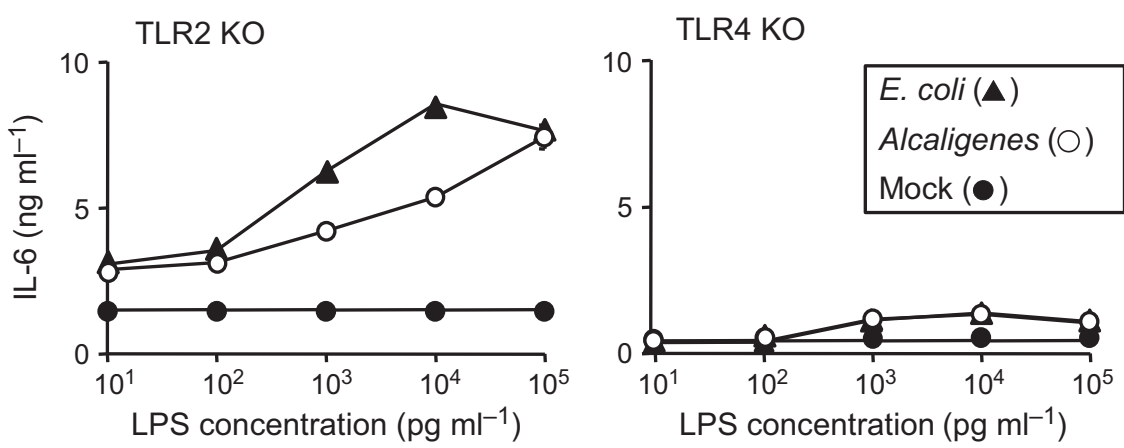

Figure 4 TLR4 recognition of Alcaligenes LPS for IL-6 production from DCs. BMDCs $\left(1 \times 10^{5}\right.$ cells per well) derived from mice lacking TLR2 (left) or TLR4 (right) were cultured with various concentrations of LPS from E. colior Alcaligenes, or were mock-treated (PBS) for $48 \mathrm{~h}$ ( $n=3 \mathrm{per}$ group). Culture supernatant was collected for the measurement of IL-6 by means of ELISA. Data are representative of two independent experiments.

Alcaligenes LPS, additive effects on IL-6 production were noted rather than inhibition (Figure 3f). These data indicate that Alcaligenes LPS does not act as a competitive inhibitor of $E$. coli LPS.

We next used TLR4-deficient mice to confirm that the LPSinduced IL-6 production was mediated by TLR4. Unlike those from wild-type mice, TLR4-deficient DCs failed to respond to both Alcaligenes- and E. coli-derived LPS, whereas TLR2deficient DCs produced comparable levels of IL-6 following LPS stimulation (Figure 4). These results suggest that both Alcaligenes- and E. coli-derived LPS act as TLR4 agonists.

\section{Alcaligenes LPS shows potent IgA-inducing activity}

Our previous study suggested that the presence of Alcaligenes in PPs, a major organized inductive tissue located in the intestinal mucosa, seems to be beneficial for the creation of an immune surveillance system by inducing and/or enhancing IgA production. ${ }^{5}$ Indeed, heat-killed Alcaligenes promoted the production of IgA-enhancing cytokines (e.g., IL-6 and APRIL). ${ }^{5}$ We found that IgA production was enhanced when $\mathrm{B}$ cells were cultured with heat-killed Alcaligenes in the presence of DCs and that this effect was reduced when the LPS was reduced by alkaline treatment (Supplementary Figure S5). 


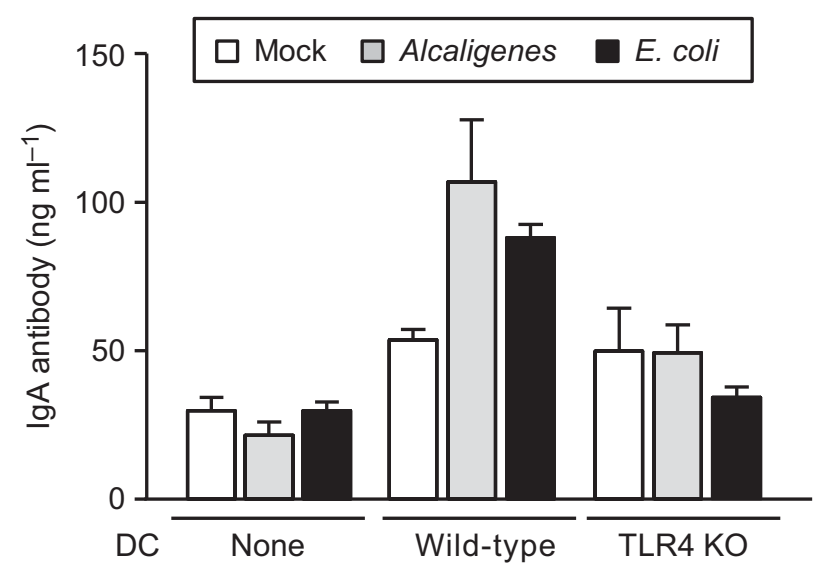

Figure 5 DC-mediated enhancement of IgA production by Alcaligenes LPS. Purified $\mathrm{B}_{220}{ }^{+} \mathrm{B}$ cells were cultured with $200 \mathrm{pg}$ of LPS from E. coli or Alcaligenes, or were mock-treated (PBS) in the presence or absence of wild-type or TLR4-deficient DCs ( $n=3$ per group). After a 5-day incubation, the culture supernatant was collected to determine the IgA concentration by means of ELISA. Data are representative of two independent experiments.

We next examined whether Alcaligenes LPS could enhance IgA production from $B$ cells. When cultures containing both $B$ cells and DCs were treated with Alcaligenes- or E. coli-derived LPS, IgA production was enhanced relative to that of the mocktreated group (Figure 5). The increase in IgA production induced by Alcaligenes LPS was reduced by treatment with an anti-IL-6-neutralizing antibody, suggesting that IL-6 is a prerequisite for the Alcaligenes LPS-induced increase in IgA production (Supplementary Figure S6). In contrast, no increase in IgA production was detected when B cells alone were cultured with either type of LPS (Figure 5). These findings suggest that the IgA-enhancing activity of LPS is dependent on DCs, which is consistent with previous studies. ${ }^{1}$ When B cells were co-cultured with TLR4- or TLR2-deficient DCs in the presence of LPS, no enhancement of IgA production was noted in the former cultures, but in the latter cultures IgA production was enhanced (Figure 5 and Supplementary Figure S7). These data collectively indicate that Alcaligenes LPS enhances IgA production through TLR4-mediated recognition by DCs.

\section{Alcaligenes LPS induces a low inflammatory reaction in vivo}

Endotoxin activity, determined by limulus amebocyte lysate test, has been correlated with inflammatory activities such as pyrogenicity and mitogenicity. ${ }^{15}$ We therefore examined the endotoxin activity of Alcaligenes LPS by using the limulus amebocyte lysate test. Alcaligenes LPS showed low endotoxin activity compared with that of E. coli LPS, which was used to represent classic, characteristic gram-negative LPS (Supplementary Figure S8). In addition, in agreement with the in vitro IL-6 production-inducing ability of Alcaligenes LPS (Figure 3b and Supplementary Figure S3), the serum IL-6 level was lower in Alcaligenes LPS-administered mice than in E. coli LPS-administered mice (Figure 6a). Accordingly, we next compared the endotoxin activity of Alcaligenes LPS and pathogenic (O111) and commensal (S17) E. coli LPS in vivo by examining symptoms such as hypothermia, lung inflammation, and leukopenia. Severe hypothermia was induced in mice that received E. coli LPS, whereas minimal changes in body temperature were noted in mice administered Alcaligenes LPS (Figure 6b). Consistent with previous reports, ${ }^{16}$ tissue damage together with massive infiltration of inflammatory cells (e.g., neutrophils and eosinophils) was observed in E. coli LPSadministered mice, whereas Alcaligenes LPS-administered mice showed few inflammatory symptoms in the lung and intestine (Figure $\mathbf{6 c}$ and unpublished data). In addition, transient leukopenia was mitigated in mice that received Alcaligenes LPS compared with those that received E. coli LPS (Figure 6d). Consistently, we found the decreased numbers of $B$ cells in the PPs (unpublished data); however, lymphocyte numbers recovered to normal levels $24 \mathrm{~h}$ after administration of Alcaligenes LPS, whereas the leukopenia remained severe in E. coli LPS-administered mice (Figure 6d). Taken together, these results demonstrate that Alcaligenes LPS exhibits biologically benign activity with lower inflammatory properties than those of E. coli LPS.

\section{Adjuvanticity of Alcaligenes LPS}

Our findings regarding the unique features of Alcaligenes LPS prompted us to explore its possible application as a vaccine adjuvant. To this end, mice were immunized subcutaneously with ovalbumin (OVA) together with Alcaligenes LPS or E. coli LPS. We also included alum as a control adjuvant. Comparable levels of OVA-specific serum IgG were detected in mice immunized with OVA plus either Alcaligenes LPS or E. coli LPS, and these levels were higher than those induced by alum (Figure 7a). Of note, alum preferentially induced OVA-specific IgG1 antibody, whereas Alcaligenes LPS and E. coli LPS induced both IgG1 and IgG2a responses, suggesting that both Th1- and Th2-type immune responses were induced (Figures $7 \mathbf{b}$ and $\mathbf{c}$ ). In addition, Alcaligenes LPS and E. coli LPS induced IL-17producing T cells (Figure 7d). Together, these findings suggest that Alcaligenes LPS can induce both humoral immune responses as well as Th1, Th2, and Th17 cells, which are similar immune responses to those induced by E. coli LPS.

\section{DISCUSSION}

Unlike other peripheral lymphoid tissues, PPs are continuously exposed to biological stimulation from numerous pathological and beneficial antigens including commensal bacteria, which gives rise to a homeostatic inflammatory condition that results from the harmonizing balancing act between immune surveillance and elimination, and to symbiotic conditions without causing excessive inflammation. ${ }^{17}$ Accumulating evidence suggests that mucosal IgA and innate lymphoid cells are critical components for the creation and maintenance of the balanced homeostatic composition of commensal bacteria. ${ }^{18,19}$ We previously reported that Bordetella, one of the LRC bacteria that resides in PPs, induces the production of several cytokines (e.g., IL-1 $\beta$, IL-6, IL-10, IL-12p40, and IL-23) by DCs. ${ }^{6}$ The production of these cytokines subsequently promotes an type 3 
a

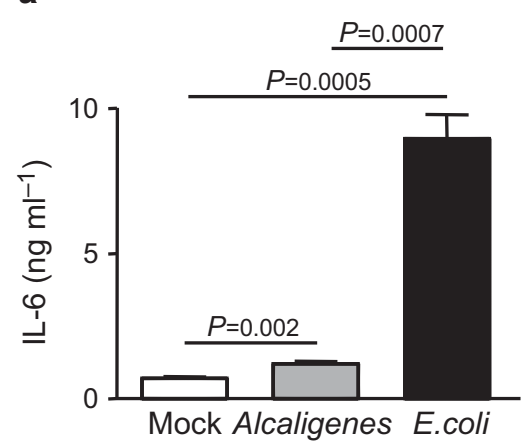

b

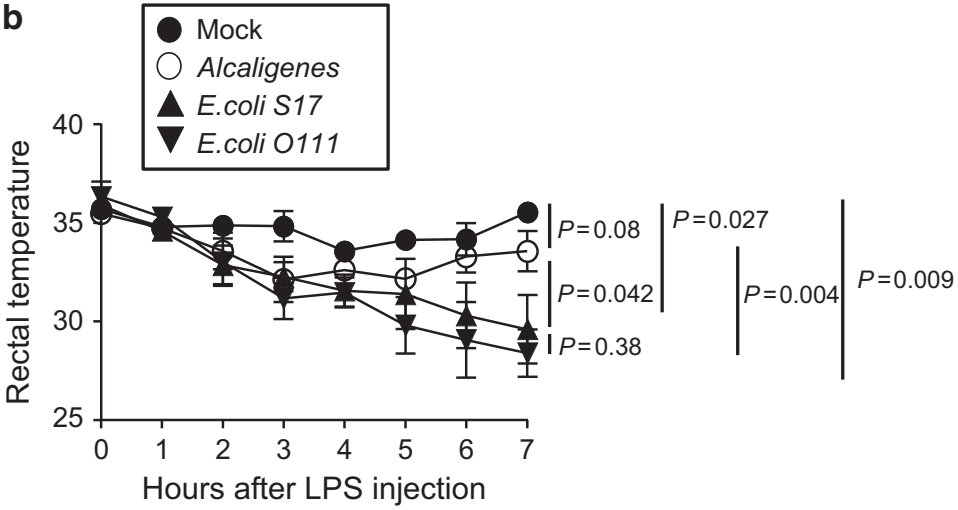

C

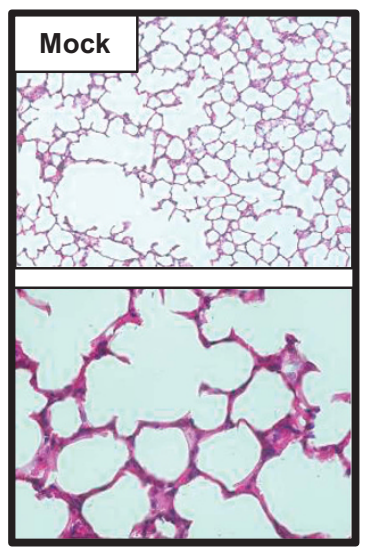

d 6 hours
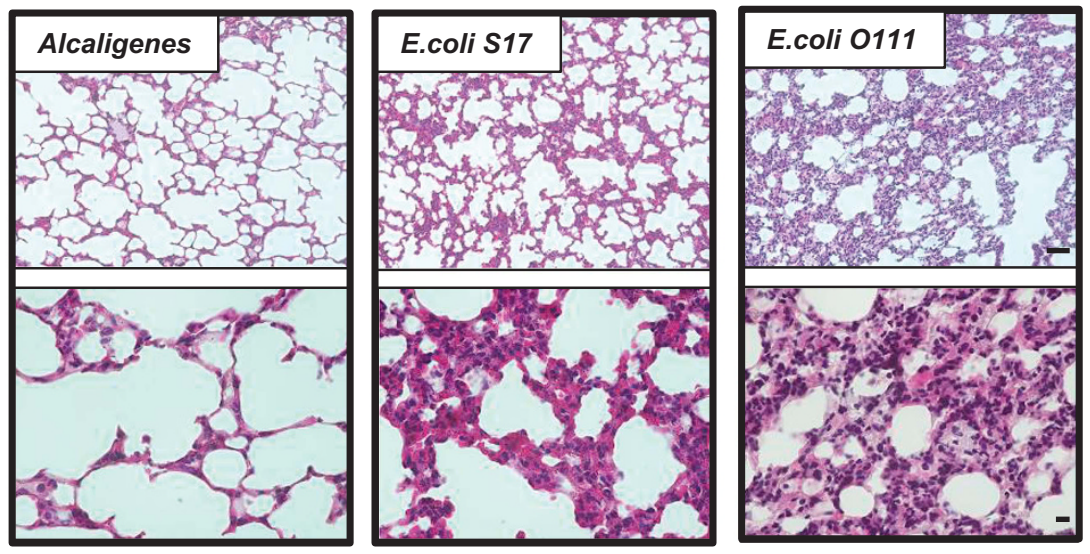

24 hours
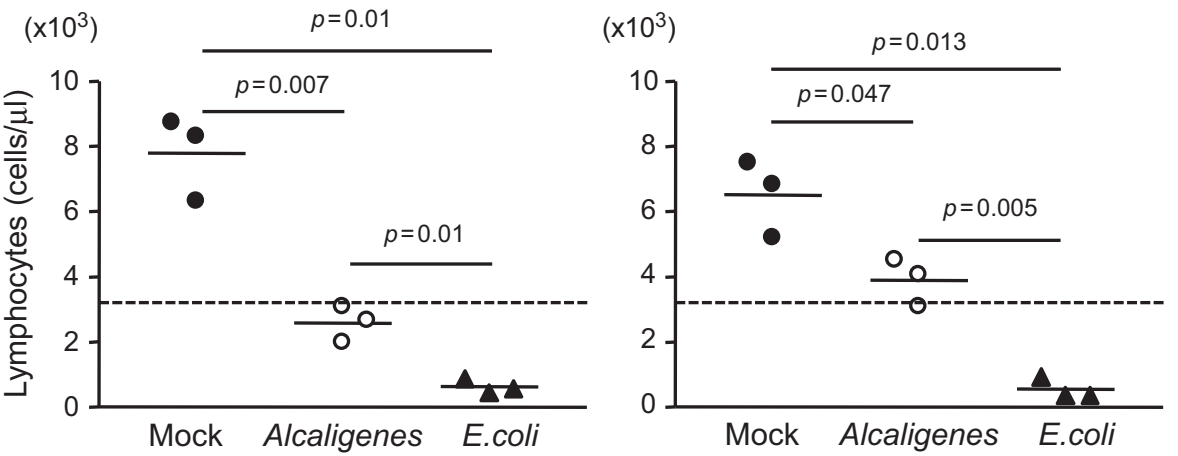

Figure 6 Alcaligenes LPS induces a limited inflammatory response in vivo. Mice were intraperitoneally injected with $1.5 \mathrm{mg}$ of LPS from E. coli or Alcaligenes or were mock-treated (PBS) ( $n=3$ per group). (a-c) (a) Seven hours after LPS injection, serum was collected to determine the IL-6 concentration by means of ELISA. (b) Rectal temperature was measured every hour for $7 \mathrm{~h}$. (c) Seven hours after LPS injection, lung sections were obtained and stained with hematoxylin and eosin. Scale bar: $50 \mu \mathrm{m}$ (upper) and $10 \mu \mathrm{m}$ (bottom). (d) Mice were subcutaneously injected with $100 \mu \mathrm{g}$ of LPS from E. coli or Alcaligenes or were mock-treated (PBS) ( $n=3$ per group). Six and $24 \mathrm{~h}$ after LPS injection, the number of lymphocyte in the blood was determined by using Vest scan HMII. Data are representative of two independent experiments.

innate lymphoid cells-mediated IL-22 response and limits the development of pro-inflammatory Th17-mediated responses. ${ }^{6}$ Among these cytokines, the production of IL- $1 \beta$, IL-10, and IL23 was decreased after exposure to heat-killed LRC bacteria compared with live LRC bacteria, whereas IL-6 and IL-12p40 were induced by both live and killed LRC bacteria. ${ }^{6}$ The later finding is consistent with our original finding. ${ }^{5}$ Constitutive colonization of DCs by LRC bacteria thus maintains the expression of viability-dependent cytokines, including IL-1 $\beta$,
IL-10, and IL-23. Our current findings revealed that IL-6 is induced by heat-killed Alcaligenes and Alcaligenes LPS.

Our current study demonstrated that IgA production was enhanced by Alcaligenes LPS in vitro. Our preliminary experiments further indicated that oral administration of Alcaligenes LPS into germ-free mice resulted in the promoted IgA responses in the intestine (unpublished data). Further, nasal vaccination with antigen and Alcaligenes LPS resulted in the induction of antigen-specific IgA responses, whereas 
a

OVA-specific lgG

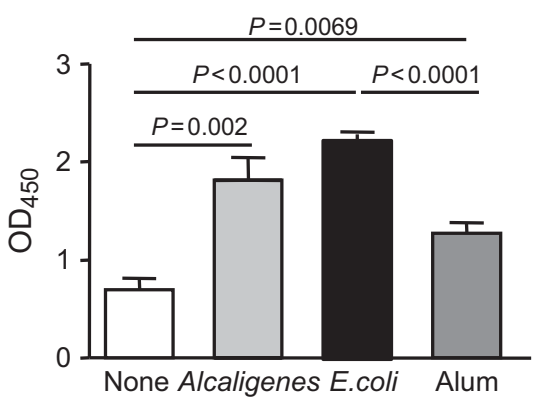

C

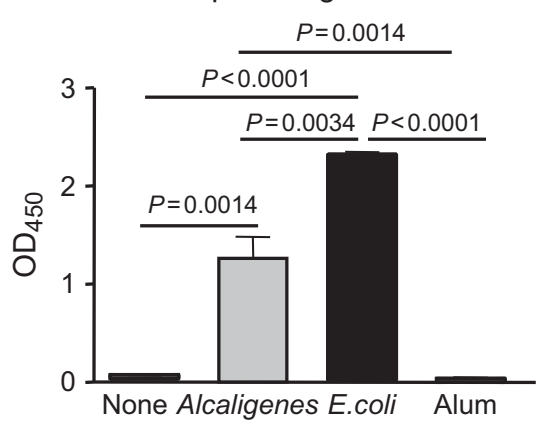

b

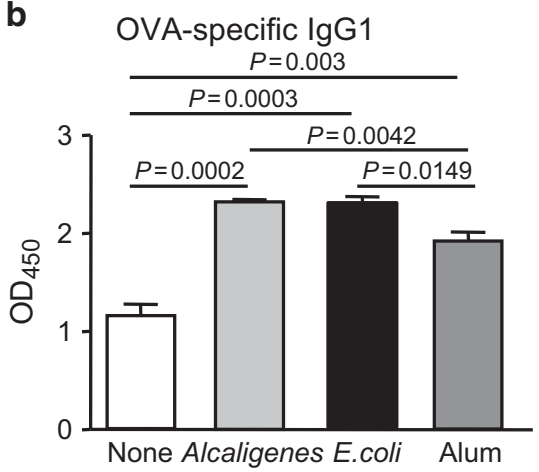

d

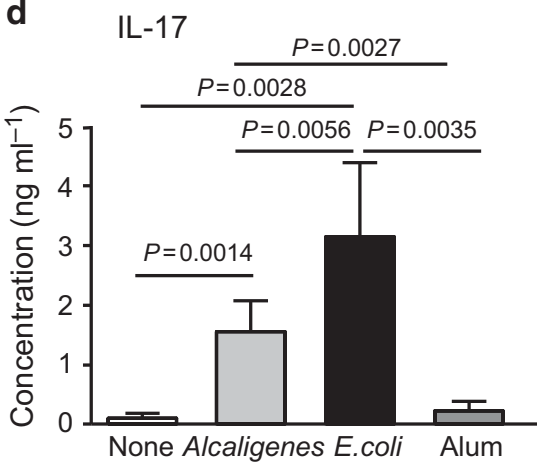

Figure 7 Adjuvanticity of Alcaligenes LPS to induce antigen-specific immune responses. Mice were subcutaneously immunized with OVA plus LPS or alum. (a-c) Serum was collected to evaluate levels of OVA-specific lgG (a), IgG1 (b), and IgG2a (c) by using an ELISA. (d) Splenic CD4 ${ }^{+}$T cells were cultured with OVA in the presence of DCs. IL-17 production in the culture supernatant was assessed. Data are representative of two independent experiments ( $n=4$ per group).

systemic vaccination induced antigen-specific IgG but not IgA antibody responses (unpublished data). These findings demonstrated Alcaligenes LPS is an effective adjuvant for supporting antigen-specific humoral immunity. Although the link between IL-6 and IgA production is controversial, ${ }^{20-22}$ our current findings demonstrated that Alcaligenes and its components (specifically LPS) induce IgA production through an IL-6dependent mechanism. In contrast, heat-killed Alcaligenes and Alcaligenes LPS induced very little IL-10 production. Our previous study demonstrated that other IgA-promoting factors, such as TGF- $\beta$ and BAFF, are induced by heat-killed Alcaligenes, ${ }^{5}$ but these factors were barely enhanced by Alcaligenes LPS and consequently no IgA production was noted when $\operatorname{IgA}^{-} \operatorname{IgM}^{+} \mathrm{B}$ cells were cultured under the same experimental conditions (unpublished data). Therefore, it is plausible that IL-6-mediated enhancement of IgA production from IgA-switched B cells is a pathway in the Alcaligenes LPSmediated IgA enhancement.

In addition to LPS, other microbial components may be involved in the enhancement of IgA class switching and production. In this context, we found that, unlike LPS-mediated enhancement of IgA production, IgA production was still increased by heat-killed Alcaligenes in the absence of TLR4 (unpublished data). It has been reported that cooperative signaling by LPS and lipoproteins, which activate TLR4 and TLR2, respectively, is necessary for the efficient induction of IL-10. ${ }^{23,24}$ Given that lipoprotein genes are universally distributed in gram-negative bacteria, ${ }^{25}$ it may be that Alcaligenes lipoproteins are needed to induce the production of IL-10 and other cytokines.

IgA is the most abundant class of antibody in the intestinal lumen and is reported to prevent pathogenic infection through its neutralizing properties, blocking toxins and pathogens from adhering to the intestinal epithelium. ${ }^{1}$ IgA also appears to help maintain the homeostatic composition of commensal bacteria, because up to $70 \%$ of commensal bacteria in the gut lumen are coated with $\operatorname{IgA}$, and this association is instrumental to the selective transport of bacteria into PPs across M cells. ${ }^{18,26}$ In addition, the systemic antibody response against commensal bacteria is increased in IgA-deficient mice. ${ }^{27}$ The unique immunological properties of LRC bacteria (e.g., Alcaligenes and Bordetella), such as inducing cytokines (e.g., IL-23 and IL-6) that promote the type 3 innate lymphoid cells-mediated IL-22 response and enhancing IgA production, likely are critical factors in the host immune surveillance and maintenance of immunological homeostasis in the gut.

Although many gut commensal bacteria induce IL-6 production from DCs via LPS, it remains unclear why LRC bacteria (e.g., Alcaligenes and Bordetella) uniquely localize in the PPs, where they induce IgA production. In the current study, we found that the uptake efficiency of the DCs in the PPs was higher for Alcaligenes than for E. coli, suggesting that some other factors rather than the inflammatory property of LPS may be involved. In fact, we previously reported that Alcaligenes efficiently binds to some percentage of the total number of 
$\mathrm{M}$ cells located dome epithelium of PP. ${ }^{10}$ We are currently testing our hypothesis that $\mathrm{M}$ cells uniquely express receptors that recognize some ligands expressed on Alcaligenes for efficient uptake into PPs. Together with the low inflammatory property of Alcaligenes LPS, these functions allow the preferential and sustained localization of Alcaligenes in the PPs to create a harmonized immunological condition.

It is known that LPS from pathogenic bacteria has an adjuvant effect, but its toxic properties interfere with its practical use as an adjuvant. The LPS structure typically consists of a lipid A and a core oligosaccharide region (an $\mathrm{O}$-antigen region), which is a primary element for the immunestimulating activity of LPS. ${ }^{28}$ One possibility is different ratio of $\mathrm{O}$-antigen region and lipid A may determine the difference of the inflammatory activity of Alcaligenes- and E. coli LPS. Another possibility is that the structure of lipid A may be different between the two LPS. It was reported that the structure of lipid A (e.g., length and linkage of fatty acids) differs depending on the strain of gram-negative bacteria ${ }^{29}$ and the culture conditions, ${ }^{30}$ which, in turn, affects its biological activity. The lipid A component of LPS has similarly been reported to have both adjuvant and toxic properties. ${ }^{31} \mathrm{~A}$ previous study showed that chemically synthesized 3-Odeacylated monophosphoryl lipid A derived from Salmonella Minnesota Re595 LPS induced lower levels of inflammatory cytokines (e.g., TNF- $\alpha$ ) from DCs compared with the parental S. Minnesota Re595 lipid A owing to a defect in CD14dependent TLR4/MD2 dimerization that resulted in reduced production of inflammatory cytokines induced by downstream signaling through TIRAP-MyD88. ${ }^{32}$ Thus, the weak activity of Alcaligenes LPS as a TLR4 agonist raises the possibility of its use as an adjuvant and our current findings support this concept. Our current and separate study is aimed at the elucidating and comparing molecular structures of Alcaligenes LPS with those of E.coli LPS.

In conclusion, our present study identified a novel mutually beneficial relationship between hosts and LRC bacteria. Alcaligenes LPS promoted homeostasis rather than massive inflammation by potentially participating in the host immunosurveillance system through the production of IgA. LRC bacteria form niches under close contact with numerous immune cells in the GALT. The weak agonistic characteristics of Alcaligenes LPS may be necessary for Alcaligenes survival under this unique circumstance and may be a factor in its effectiveness as a vaccine adjuvant.

\section{METHODS}

Mice. BALB/c mice were purchased from Japan Clea Co. (Tokyo, Japan). CD11c-DTR tg mice (Balb/c background) were obtained from the Jackson Laboratory (Bar Harbor, ME, USA) and depleted of DCs by intraperitoneal injection with $500 \mathrm{ng}$ of DT (Sigma-Aldrich, St. Louis, MO, USA). ${ }^{11}$ Mice lacking TLR2 or TLR4 (C57BL/6 background) were purchased from Oriental Bio Service, Inc. (Kyoto, Japan). Mice were maintained under specific pathogen-free conditions at the Institute of Medical Science, the University of Tokyo. All experiments were conducted in accordance with the guidelines of the Animal Care and Use Committees of the University of Tokyo and of the National Institutes of Biomedical Innovation, Health, and Nutrition.

Bacteria culture. Alcaligenes faecalis was purchased from the National Institute of Technology and Evaluation Biological Resource Center (NBRC, $13111 \mathrm{~T}$ ) and grown in Tryptic Soy Broth (BD Diagnostics, Sparks, MD, USA) at $37^{\circ} \mathrm{C}$. GFP-Alcaligenes were generated as previously reported ${ }^{5}$ and grown at $37^{\circ} \mathrm{C}$ in Tryptic Soy Broth containing streptomycin $(50 \mu \mathrm{g} / \mathrm{ml})$ and isopropyl $\beta$-D-1-thiogalactopyranoside (1 mM). E. coli O111 (NBRC IID561) was obtained from the Pathogenic Microbes Repository Unit (Tokyo, Japan) and grown in LB Broth (Nacalai Tesque, Inc., Kyoto, Japan) at $37^{\circ} \mathrm{C}$. Bacteria were heat-killed by incubating them at $65^{\circ} \mathrm{C}$ for $30 \mathrm{~min}$. For LPS depletion, heat-killed bacteria were incubated in $0.1 \mathrm{~N} \mathrm{NaOH}$ in $95 \%$ ethyl alcohol (Nacalai Tesque, Inc.) at $30{ }^{\circ} \mathrm{C}$ for $60 \mathrm{~min} .{ }^{33}$ After this incubation, the reaction was stopped by adding an equal volume of acetic acid (WAKO Chemicals, Tokyo, Japan) in 95\% ethyl alcohol, and the neutralized solution was rapidly cooled in an ice-water bath. ${ }^{33}$

LPS preparation and the limulus test. LPS was extracted from lyophilized Alcaligenes and E. coli by using an LPS Extraction Kit (iNtRON Biotechnology, Inc., Sangdaewon-Dong, Korea). The quantity of purified LPS per cell was calculated by dividing the weight of freeze-dried LPS by the CFU of bacteria. Endotoxicity was measured by means of the limulus amebocyte lysate test using an Endospecy ES-50M kit (Seikagaku Co., Tokyo, Japan) according to the manufacturer's instructions.

Intestinal loop assay. Mice were anesthetized by using an isoflurane vaporizer. An intestinal loop containing a single $\mathrm{PP}$ was ligated and incubated with GFP-Alcaligenes $\left(5 \times 10^{8} \mathrm{CFU}\right.$ in $100 \mu \mathrm{l}$ of PBS) for 2 h. ${ }^{10}$ After this 2-h incubation, the PP was excised from the ligated intestine, washed with PBS, and then subjected to immunohistochemical analysis. To measure bacterial uptake efficacy, the PPs were incubated in $100 \mu \mathrm{g} / \mathrm{ml}$ gentamycin solution (Nacalai Tesque, Inc.) for $30 \mathrm{~min}$ at room temperature and then washed with PBS. The PPs were then homogenized with PBS and plated onto Tryptic Soy agar (BD Diagnostics) containing $50 \mu \mathrm{g} / \mathrm{ml}$ streptomycin for $16 \mathrm{~h}$ at $37^{\circ} \mathrm{C}$. Bacterial uptake efficiency was quantified by counting the number of colonies.

Immunohistochemistry. PPs were fixed in $4 \%$ paraformaldehyde for $16 \mathrm{~h}$ at $4{ }^{\circ} \mathrm{C}$, washed, and then incubated with $20 \%$ sucrose for $16 \mathrm{~h}$ at $4{ }^{\circ} \mathrm{C}$. The tissues were then embedded in OCT compound (Sakura Finetechnical Co., Tokyo, Japan). Sections were stained with appropriate antibodies and underwent the tyramide signal amplification (TSA) system (PerkinElmer, Shelton, CT, USA). ${ }^{11}$ In brief, cryostat sections $(7 \mu \mathrm{m})$ were treated with $1 \% \mathrm{H}_{2} \mathrm{O}_{2}$ and an avidin/ biotin blocking kit (Vector Laboratories, Burlingame, CA, USA) to quench endogenous peroxidase and biotin. After being blocked with anti-CD16/CD32 antibody in TNT buffer $(0.1 \mathrm{~m}$ Tris- $\mathrm{HCl}(\mathrm{pH} 7.5)$, $0.15 \mathrm{M} \mathrm{NaCl}, 0.05 \%$ Tween 20) for $15 \mathrm{~min}$ at room temperature, the sections were stained with a biotinylated anti-CD11c mAb (BD Bioscience, San Diego, CA, USA). After washing twice with TNT buffer, the sections were incubated with HRP-conjugated streptavidin (Pierce, Rockford, IL, USA) for $30 \mathrm{~min}$ at $4{ }^{\circ} \mathrm{C}$, and the fluorescent signal was amplified by using the TSA system with a Cy 5 dye. After the specimens were stained with DAPI (Sigma-Aldrich), they were analyzed by using a confocal laser-scanning microscope (TCS SP2; Leica, Wetzlar, Germany).

Whole-mount fluorescein in situ hybridization (FISH) analysis. To detect Alcaligenes, oligonucleotide probes for ALBO, which detect position 699-716 in the $23 \mathrm{~S}$ rRNA of Alcaligenes spp., were purchased from Invitrogen-Molecular Probes (Carlsbad, CA, USA). Isolated tissue segments were fixed in $4 \%$ PFA for $16 \mathrm{~h}$ at $4{ }^{\circ} \mathrm{C}$ and then washed with PBS. Tissues were hybridized for $16 \mathrm{~h}$ in hybridization buffer $(0.9 \mathrm{M} \mathrm{NaCl}, 20 \mathrm{~mm}$ Tris- $\mathrm{HCl}, 45 \%$ formamide, $0.1 \%$ SDS, and 
$10 \mu \mathrm{g} / \mathrm{ml}$ DNA probe) at $60^{\circ} \mathrm{C}$. After being washed twice in washing buffer $(0.45 \mathrm{M} \mathrm{NaCl}, 20 \mathrm{~mm}$ Tris- $\mathrm{HCl}, 45 \%$ formamide, and $0.01 \%$ SDS $)$ for $10 \mathrm{~min}$ at $60^{\circ} \mathrm{C}$, the tissue segments were flushed with PBS. To detect the epithelial layer, the specimen was stained with Alexa Fluor 633-labeled wheat-germ agglutinin (WGA, $10 \mu \mathrm{g} / \mathrm{ml}$, InvitrogenMolecular Probes) for $1 \mathrm{~h}$. After being washed with PBS, the tissue samples were mounted and examined by using a confocal laserscanning microscope (TCS SP2; Leica); the fluorescent dots in each dome were counted.

\section{Generation of bone marrow-derived DCs and isolation of DCs from} PPs. Cells were isolated from the PPs and BM as previously described. ${ }^{34,35}$ Briefly, PPs were stirred in $1.5 \mathrm{mg} / \mathrm{ml}$ collagenase (Wako Chemicals) to obtain a single-cell suspension. Bone marrow cells were flushed from femurs by using sterile RPMI 1640 medium and treated with red blood cell lysis buffer $\left(1.5 \mathrm{M} \mathrm{NH}_{4} \mathrm{Cl}, 100 \mathrm{mM}\right.$ $\mathrm{KHCO}_{3}$ and $10 \mathrm{~mm}$ EDTA-2Na) for $5 \mathrm{~min}$, then quickly washed with RPMI 1640 medium. Cells were re-suspended with pre-warmed RPMI 1640 containing GM-CSF. Half of the DC growth media was replaced with fresh RPMI 1640 containing GM-CSF every 2 days. DCs were purified from cells isolated from the PPs or BM cells cultured with GMCSF for 6 days by using anti-mouse CD11c magnetic beads and a Magnetic Cell Separation System (MACS; Miltenyi Biotech, Bergisch Gladbach, Germany) according to the manufacturer's protocols.

DC culture and $\mathrm{ED}_{50}$ value calculations. BMDCs or DCs isolated from PPs $\left(1 \times 10^{5}\right.$ cells) were cultured with various concentrations of LPS or heat-killed bacteria. After $48 \mathrm{~h}$ of incubation, cells and culture supernatant were collected for the analysis of cell viability by trypan blue exclusion and for the measurement of IL-6, TNF- $\alpha$, and IL-10 production by means of ELISA (R\&D Systems, Minneapolis, MN, USA), respectively. IL- $6 \mathrm{ED}_{50}$ values were calculated from IL-6 production vs. log concentration curves by using the sigmoidal curves of Prism computer software (GraphPad Software, San Diego, CA, USA). ${ }^{11}$

In vitro IgA production assay. PP cells were isolated as previously described. ${ }^{34}$ Purified B220 ${ }^{+}$cells $\left(2 \times 10^{5}\right.$ cells $)$ were cultured with or without BMDCs $\left(1 \times 10^{5}\right.$ cells $)$ in the presence of $200 \mathrm{pg}$ of LPS from E. coli or Alcaligenes, or were mock-treated (PBS). After a 5-day incubation, the culture supernatant was collected to determine the IgA concentration by means of ELISA as previously described. ${ }^{36}$

LPS-induced inflammation. Mice were injected intraperitoneally with LPS ( $1.5 \mathrm{mg} /$ body), and rectal temperature was monitored every hour. Serum was collected $7 \mathrm{~h}$ after the LPS injection and used to measure IL-6 levels by means of ELISA (R\&D Systems). Simultaneously, lung tissues were collected and fixed in $4 \%$ paraformaldehyde for $16 \mathrm{~h}$ and embedded in paraffin for immunohistochemical analysis by staining with hematoxylin and eosin (WAKO Chemicals). For the analysis of leukopenia, mice were subcutaneously injected with $100 \mu \mathrm{g}$ of LPS, and the number of lymphocytes in the blood was determined by using Vet Scan HMII (Abaxis, Union City, CA, USA).

Immunization and detection of OVA-specific antibodies and IL-17 production from T cells. Mice were subcutaneously immunized with $10 \mu \mathrm{g}$ of OVA plus $100 \mu \mathrm{g}$ of LPS or $4 \mathrm{mg}$ of alum (Thermo Fisher Scientific Inc., Waltham, Massachusetts, USA) on days 0 and 7. On day 14 , serum was collected and tested for OVA-specific antibodies by means of an ELISA as previously reported. ${ }^{37}$ Simultaneously, spleen cells were collected to analyze IL-17 production from T cells. Briefly, splenic $\mathrm{CD} 4{ }^{+}$cells were purified by using anti-mouse CD4 magnetic beads and a MACS System (Miltenyi Biotech). 30 Gy-irradiated splenic cells were used as antigen-presenting cells (APCs). The $\mathrm{CD}^{+}{ }^{+}$cells $\left(2 \times 10^{5}\right.$ cells $)$ were cultured with the APCs $\left(2 \times 10^{4}\right.$ cells $)$ in the presence of $1 \mathrm{mg} / \mathrm{ml}$ OVA. After a 4 -day incubation, the culture supernatant was collected to determine the IL-17 A concentration by using a BD cytometric bead array kit (BD Biosciences) according to the manufacturer's instructions.
Statistical analyses. The results were compared by using the Student's $t$-test. Statistical significance was established at $P<0.05$.

SUPPLEMENTARY MATERIAL is linked to the online version of the paper at http://www.nature.com/mi

\section{ACKNOWLEDGMENTS}

This work was supported by grants from the Core Research for Evolutional Science and Technology Program of the Japan Agency for Medical Research and Development (H.K.); by the Ministry of Education, Culture, Sports, Science, and Technology of Japan (Grants-in-Aid for Scientific Research S (H.K.; 23229004), for Scientific Research B (J.K.; 26293111), for Scientific Research on Innovative Areas (J.K., 16H01373), for Scientific Research on Innovative Areas in Middle Molecular Strategy (K.F.), for Scientific Research A (K.F.; JP15H05836), for Scientific Research C (A.S.), for Scientific Research B (Y.F.; J26282211), for Young Scientists B (N.S.; 17K17686), and for a JSPS Research Fellow (N.S.; 17J07480)); by the JSPS NEXT program (Y.F.; LR025); by the Practical Research Project for Allergic Diseases and Immunology (Research on Allergic Diseases and Immunology) and for the Research on Development of New Drugs (Adjuvant database project) from the Japan Agency for Medical Research and Development (AMED; J.K., K.J.I., and H.K.) and by the Ministry of Health, Labour and Welfare (MHLW) (J.K.); by the Science and Technology Research Promotion Program for Agriculture, Forestry, Fisheries, and Food Industry (J.K.) by the Grant for Joint Research Project of the Institute of Medical Science, the University of Tokyo (J.K.); and by the Astellas Foundation for Research on Metabolic Disorders (J.K.), the Public Health Research Foundation for Public Health Science (N.S.), the Naito Foundation (H.M.), the Terumo Foundation for Life Sciences and Arts (J.K.), the Suzuken Memorial Foundation (J.K.), and the Canon Foundation (J.K.). This work was collaborated with Astellas Pharma Inc. for Expansion of the Scope of Collaborative Research Utilizing Rice-Based Oral Vaccine "MucoRice" Technology (H.K.).

\section{AUTHOR CONTRIBUTIONS}

NS designed the study, performed experiments, analyzed data, and wrote the manuscript; JK designed the study, supervised, analyzed data, and wrote the manuscript; YF performed limulus test and wrote the manuscript; $\mathrm{KM}, \mathrm{NK}$, and AS prepared LPS and performed limulus test; HM provided samples; SS supervised the research; $\mathrm{KH}$, NK, and KJI performed leukopenia; KF supervised the research and wrote the manuscript; HK designed the study, supervised, analyzed data, and wrote the manuscript.

\section{DISCLOSURE}

S.S. is an employee of The Research Foundation for Microbial Diseases of Osaka University. The other authors declared no conflict of interest.

c) 2018 Society for Mucosal Immunology

\section{REFERENCES}

1. Fagarasan, S., Kawamoto, S., Kanagawa, O. \& Suzuki, K. Adaptive immune regulation in the gut: T cell-dependent and T cell-independent IgA synthesis. Annu. Rev. Immunol. 28, 243-273 (2010).

2. Umesaki, Y., Setoyama, H., Matsumoto, S. \& Okada, Y. Expansion of $\alpha \beta$ T-cell receptor-bearing intestinal intraepithelial lymphocytes after microbial colonization in germ-free mice and its independence from thymus. Immunology 79, 32-37 (1993).

3. Ivanov, II et al. Induction of intestinal Th17 cells by segmented filamentous bacteria. Cell 139, 485-498 (2009).

4. Atarashi, K. et al. Induction of colonic regulatory $\mathrm{T}$ cells by indigenous Clostridium species. Science 331, 337-341 (2011).

5. Obata, T. et al. Indigenous opportunistic bacteria inhabit mammalian gutassociated lymphoid tissues and share a mucosal antibody-mediated symbiosis. Proc. Natl. Acad. Sci. USA 107, 7419-7424 (2010).

6. Fung, T.C. et al. Lymphoid-tissue-resident commensal bacteria promote members of the IL-10 cytokine family to establish mutualism. Immunity 44, 634-646 (2016). 
7. Sonnenberg, G.F. et al. Innate lymphoid cells promote anatomical containment of lymphoid-resident commensal bacteria. Science 336, 1321-1325 (2012).

8. Pulendran, B. et al. Lipopolysaccharides from distinct pathogens induce different classes of immune responses in vivo. J. Immunol. 167, 50675076 (2001).

9. Park, B.S. et al. The structural basis of lipopolysaccharide recognition by the TLR4-MD-2 complex. Nature 458, 1191-1195 (2009).

10. Sato, S. et al. Transcription factor Spi-B-dependent and -independent pathways for the development of Peyer's patch M cells. Mucosal. Immunol 6, 838-846 (2013).

11. Obata, T. et al. Critical role of dendritic cells in $\mathrm{T}$ cell retention in the interfollicular region of Peyer's patches. J. Immunol. 191, 942948 (2013).

12. de Waal Malefyt, R., Abrams, J., Bennett, B., Figdor, C.G. \& de Vries, J.E. Interleukin 10 (IL-10) inhibits cytokine synthesis by human monocytes: an autoregulatory role of IL-10 produced by monocytes. J. Exp. Med. 174, 1209-1220 (1991).

13. Kutuzova, G.D., Albrecht, R.M., Erickson, C.M. \& Qureshi, N. Diphosphoryl lipid A from Rhodobacter sphaeroides blocks the binding and internalization of lipopolysaccharide in RAW 264.7 cells. J. Immunol. 167, 482-489 (2001).

14. Yoshimura, A., Kaneko, T., Kato, Y., Golenbock, D.T. \& Hara, Y. Lipopolysaccharides from periodontopathic bacteria Porphyromonas gingivalis and Capnocytophaga ochracea are antagonists for human toll-like receptor 4. Infect. Immun. 70, 218-225 (2002).

15. Elin, R.J., Sandberg, A.L. \& Rosentreich, D.L. Comparison of the pyrogenicity, Limulus activity mitogenicity and complement reactivity of several bacterial endotoxins and related compounds. J. Immunol. 117, 1238-1242 (1976).

16. Larsson, R., Rocksen, D., Lilliehook, B., Jonsson, A. \& Bucht, A. Dosedependent activation of lymphocytes in endotoxin-induced airway inflammation. Infect. Immun. 68, 6962-6969 (2000).

17. Hooper, L.V. \& Gordon, J.I. Commensal host-bacterial relationships in the gut. Science 292, 1115-1118 (2001).

18. Mathias, A., Pais, B., Favre, L., Benyacoub, J. \& Corthesy, B. Role of secretory IgA in the mucosal sensing of commensal bacteria. Gut Microbes 5, 688-695 (2014).

19. Guo, X. et al. Innate lymphoid cells control early colonization resistance against intestinal pathogens through ID2-dependent regulation of the microbiota. Immunity 42, 731-743 (2015).

20. Beagley, K.W. et al. Interleukins and IgA synthesis. Human and murine interleukin 6 induce high rate IgA secretion in IgA-committed B cells. J. Exp. Med. 169, 2133-2148 (1989).

21. Ramsay, A.J. et al. The role of interleukin-6 in mucosal IgA antibody responses in vivo. Science 264, 561-563 (1994).
22. Bromander, A.K., Ekman, L., Kopf, M., Nedrud, J.G. \& Lycke, N.Y. IL-6deficient mice exhibit normal mucosal IgA responses to local immunizations and Helicobacter felis infection. J. Immunol. 156, 42904297 (1996).

23. Hirata, N. et al. Selective synergy in anti-inflammatory cytokine production upon cooperated signaling via TLR4 and TLR2 in murine conventional dendritic cells. Mol. Immunol. 45, 2734-2742 (2008).

24. Schenk, M., Belisle, J.T. \& Modlin, R.L. TLR2 looks at lipoproteins. Immunity 31, 847-849 (2009).

25. Nakayama, H., Kurokawa, K. \& Lee, B.L. Lipoproteins in bacteria: structures and biosynthetic pathways. FEBS J. 279, 4247-4268 (2012).

26. van der Waaij, L.A., Limburg, P.C., Mesander, G. \& van der Waaij, D. In vivo IgA coating of anaerobic bacteria in human faeces. Gut 38, 348-354 (1996).

27. Sait, L.C. et al. Secretory antibodies reduce systemic antibody responses against the gastrointestinal commensal flora. Int. Immunol. 19, 257-265 (2007).

28. Erridge, C., Bennett-Guerrero, E. \& Poxton, I.R. Structure and function of lipopolysaccharides. Microbes. Infect. 4, 837-851 (2002).

29. Ogawa, T. et al. Immunobiological activities of chemically defined lipid A from Helicobacter pylori LPS in comparison with Porphyromonas gingivalis lipid A and Escherichia coli-type synthetic lipid A (compound 506). Vaccine 15, 1598-1605 (1997).

30. Kawahara, K., Tsukano, H., Watanabe, H., Lindner, B. \& Matsuura, M. Modification of the structure and activity of lipid $\mathrm{A}$ in Yersinia pestis lipopolysaccharide by growth temperature. Infect. Immun. 70, 4092-4098 (2002).

31. Persing, D.H. et al. Taking toll: lipid A mimetics as adjuvants and immunomodulators. Trends. Microbiol. 10, S32-S37 (2002).

32. Tanimura, N. et al. The attenuated inflammation of MPL is due to the lack of CD14-dependent tight dimerization of the TLR4/MD2 complex at the plasma membrane. Int. Immunol. 26, 307-314 (2014).

33. Niwa, M., Milner, K.C., Ribi, E. \& Rudbach, J.A. Alteration of physical, chemical, and biological properties of endotoxin by treatment with mild alkali. J. Bacteriol. 97, 1069-1077 (1969).

34. Kunisawa, J. et al. Microbe-dependent $\mathrm{CD}_{11} \mathrm{~b}^{+} \lg \mathrm{A}^{+}$plasma cells mediate robust early-phase intestinal IgA responses in mice.. Nat. Commun. 4, 1772 (2013).

35. Sato, A., Linehan, M.M. \& Iwasaki, A. Dual recognition of herpes simplex viruses by TLR2 and TLR9 in dendritic cells. Proc. Natl. Acad. Sci. USA 103, 17343-17348 (2006).

36. Kotani, Y. et al. Role of Lactobacillus pentosus Strain b240 and the Toll-like receptor 2 axis in Peyer's patch dendritic cell-mediated immunoglobulin A enhancement. PLoS ONE 9, e91857 (2014).

37. Kunisawa, J. et al. Mode of bioenergetic metabolism during B cell differentiation in the intestine determines the distinct requirement for vitamin B1. Cell Rep. 13, 122-131 (2015). 